\title{
Image Analysis of Oxidative and Glycolytic Muscle Fibers During Reperfusion Injury by Segmentation Based on Regions
}

\author{
Análisis de Imágenes de Fibras Musculares Oxidativas y Glucolíticas \\ Durante la Reperfusión Mediante Segmentación Basada en Regiones
}

\author{
Doris Haydee Rosero-Salazar ${ }^{*, * *}$
}

\begin{abstract}
ROSERO-SALAZAR, D. H. Image analysis of oxidative and glycolytic muscle fibers during reperfusion injury by segmentation based on regions. Int. J. Morphol., 34(1):127-135, 2016.

SUMMARY: Different situations cause ischemia and reperfusion injury, affecting tissues under the level of compression. In this research, abnormal characteristics in distribution of muscle fibers types in soleus and extensor carpi radialis longus, during short periods of ischemia and short and long periods of reperfusion, were determined. Fibers were classified by enzyme histochemistry techniques NADH-TR and myosin-ATPase. Measurements of areas were carried out through semiautomatic image processing by using segmentation based on regions, which evidenced significant changes in distribution during reperfusion followed to one and three hours of ischemia, as well as in comparisons of areas for all periods of reperfusion. This study strengthens the evidence about using practical procedures of image analysis in the diagnosis of tisular abnormalities.
\end{abstract}

KEY WORDS: Histochemistry; Ischemia; Reperfusion; Image analysis; Segmentation.

\section{INTRODUCTION}

Muscle fibers and extracellular intramuscular connective tissue are the main components of skeletal striated muscle. In order to identify types of fibers, the enzymatic activity is one of the characteristics that had permitted to classify myocytes according to their metabolism (oxidative, intermediate and glycolytic), velocity of contraction (slow twitch fibers, intermediate fibers and fast twitch fibers) and to molecular level, isoforms of myosin heavy chain (MHC-1, MHC-IIA, MCHIIB) (Purslow, 2010; de Freitas et al., 2009; Scott et al., 2001). Enzyme histochemistry techniques such as mATPase (myosin adenosine triphosphatase) and NADH-TR (nicotinamide adenine dinucleotide tetrazolium reductase), indicate in striated skeletal muscle tissue, fast twitch fibers that are associated with glycolytic and glycolytic-intermediate metabolism, while slow twitch fibers with oxidative metabolism (Vivo et al., 2004; Hebling et al., 2006; CarmoAraújo et al., 2007; de Freitas et al.).

Modern image analysis techniques are useful in diagnostic and investigation, especially when practical procedures as semi-automatic measurements is used to optimize the analysis through segmentation and determination of regions of interest; in this case, skeletal muscles contain a high proportion of a particular fiber type, aspect that depends on their function and localization; for example, the predominance of extensor muscles is of IIb fiber types, whereas flexor muscles is of oxidative fibers (Lexell et al., 1994; Soukup et al., 2002). The distribution of each type of fiber corresponds to the region that is of interest to be measured.

Studies conducted by Walters et al. (2008), demonstrated that skeletal muscles tolerate periods of ischemia up to six hours and the contributing factor in the degree of injury and regeneration, is the predominant fiber type. Ischemia less than six hours occur frequently in two situations, the first, relates to orthopedic surgery, including arthroscopic knee, ankle and wrist, where the interval of time for these procedures is between $15 \mathrm{~min}$ to $60 \mathrm{~min}$; the use of a pneumatic tourniquet is necessary in these surgical treatments (Hocker et al., 2013). The other condition occurs

\footnotetext{
* Magister in Biomedical Sciences. Professor of Histology. Department of Morphology. Faculty of Health. Universidad del Valle, Cali, Colombia.

${ }^{* *}$ Professor of Histology. Department of Medical Basic Sciences. Universidad Icesi, Cali, Colombia.
} 
in traumatic injuries that occur in remote areas, where access to medical care, could take at least three hours of travel; in these situations a manual tourniquet is useful. In both cases, tissues located below the level of compression suffer the effects of ischemia followed by consequences of reperfusion. One of those complications is the "non-capillary reflux syndrome", which leads to increase the ischemic condition by obstruction and oxidative stress during reperfusion. Silveira et al. (2003), observed by image analysis of microcirculation, in the initial stages of reperfusion after four hours of induced ischemia by arterial occlusion, a decreased in capillary flow rate with vasodilation and hyperemia.

In this research, abnormal patterns in the distribution of muscle fibers during reperfusion, as well as histochemical and morphometric changes in glycolytic and oxidative fibers, in soleus and extensor carpi radialis longus that underwent short periods of ischemia of one and three hours, and reperfusion from zero hours to $32 \mathrm{~d}$, were determined by using semiautomatic image analysis techniques. This work is a model to explore the use of segmentation by regions as a tool for identifying tisular changes, for this case, skeletal striated muscle tissue that underwent ischemia and reperfusion.

\section{MATERIAL AND METHOD}

Animals. Forty-two male Wistar rats from the bioterium of Universidad del Valle, were housed, fed food and water ad libitum and maintained in accordance to Institutional Standard Guide to the Use of Laboratory Animals. The Animal Ethics Committee of Universidad del Valle approved protocols used in this study.

Induced ischemia. Rats were assigned to one control group and twenty experimental groups, conformed by ischemia of one hour followed by reperfusion of zero hours, one (1) hour, 16 h, 24 h, 2 d (48 h), 4 d (96 h), 8 d (192 h), 16 d (384 h), $24 \mathrm{~d}(576 \mathrm{~h})$ and $32 \mathrm{~d}(768 \mathrm{~h})$; and ischemia of three hours followed by the same periods of reperfusion. Under anesthetic condition two pneumatic tourniquets were used, one in the right forelimb and the other in the left hindlimb. Capillary irrigation is not present at $230 \pm 20 \mathrm{mmHg}$ (Corona \& Rathbone, 2014; Hocker et al.; Walters et al.; Woitaske \& McCarter, 1998) so, established pressure of compression was $270 \mathrm{mmHg}$ during one (1) or three (3) hours. After this procedure, postoperative analgesia were provided to all animals.

Tissue samples. Euthanasia was performed once each period of reperfusion finished. Soleus and extensor carpi radialis longus were dissected, fixed by freezing in liquid nitrogen and stored at $-70^{\circ} \mathrm{C}$. Samples were cut on cryostat at $-20^{\circ} \mathrm{C}$, then, were stained for NADH-TR and mATPase $\mathrm{pH} 4.6$.

Histochemistry. NADH is a dehydrogenase enzyme found in the inner mitochondrial membrane and it is part of the electron transport chain, in which the NAD+ is reduced to form NADH. The application of NADH-TR method allows determining fibers metabolically different from each other, thus, fibers using oxidative metabolism have a greater blue reaction. This technique is useful to identify variations in the structure, organization of fibers and changes in the diameter in atrophic fibers (Hebling et al., 2009). To carry out quantitative enzyme histochemistry for NADH-TR, incubation of samples was performed during $45 \mathrm{~min}$ at 37 ${ }^{\circ} \mathrm{C}$ in Tampon Tris $0.2 \mathrm{M}$ solution (trishidroximetilaminometano $0.3 \mathrm{~g}$ and $0.1 \mathrm{M} \mathrm{HCl}) 10 \mathrm{~mL}$, with nitroblue tetrazolium (Sigma Aldrich) $10 \mathrm{mg}$, and Bnicotinamide dinucleotide $8 \mathrm{mg}$, $\mathrm{pH} 7.4$; then sampled were checked and rinsed with distilled water during one minute. These solutions were prepared freshly before incubation. Tissues were mounted in glycerol jelly medium.

Myosin heavy chains by crossover processes with actin filaments, allow determining the shortening speed according to $\mathrm{pH}$ variations in the rupture of ATP by mATPase; so that, histochemical analysis of myosin ATPase activity differentiates between fast twitch muscle fibers and slow twitch (Gueguen et al., 2005). This characteristic makes possible establishing two groups, the first, is stable acid / alkaline weak, or slow twitch fibers, in which at $\mathrm{pH}$ alkaline is observe a pale reactivity in these fibers. The other group is weak acid / alkaline-stable or fast twitch fibers that become negative ATPase faster when acidity of the pre-incubation medium is increased. The fast twitch fibers hydrolyze ATP faster than slow twitch and have greater reactivity in the presence of a basic $\mathrm{pH}$, contrary to acidic pre-incubations in which slow-twitch fibers present a more intensity of reaction (Kalmar et al., 2012; Latorre et al., 1993).

For m-ATPase $\mathrm{pH} 4.6$, tissues were pre-incubated during $25 \mathrm{~min}$ at $22^{\circ} \mathrm{C}$ in ATP I stock solution (sodium barbital $0.075 \mathrm{M}$, calcium chloride $0.1 \mathrm{M}$ and sodium acetate $0.07 \mathrm{M}$ ). After that, incubation in ATP II solution prepared with Adenosine 5'triphosphate $15 \mathrm{mg}$ (SigmaAldrich) and ATP I stock solution $10 \mathrm{~mL}$, during $40 \mathrm{~min}$ at $37{ }^{\circ} \mathrm{C}$. Then, samples were immersed twice in calcium chloride $0.1 \mathrm{M}$, each during $5 \mathrm{~min}$, later in cobalt chloride $2 \%$ during $5 \mathrm{~min}$ and rinsed with distilled water to be put in ammonium sulfide during $15 \mathrm{~s}$ to reveal. Glycerol jelly was the mounting medium. 
Image processing and morphometric analysis. Images at 10x magnification were acquired by using a DM750 microscope (Leica Microsystems), a digital camera DFC295 (Leica Microsystems), both integrated to software LASV3.8 (Leica Application Suite). These equipments located in Department of Morphology, Faculty of Health, and Universidad del Valle. The protocol for acquisition of images automatically included light exposure, low light intensity, brightness of $50 \%$, saturation of 1.1 , and gamma of 0.7 . Images retained their original characteristics of acquisition for morphometric analysis; improvements or modifications of images were not made. The software used for image analysis was Image Pro Plus version 7.0 (Media Cybernetics Inc.). For proper measurements data of images, calibrations were applied by using a standardized Stage micrometer with a scale from zero (0) to 1000 microns ( $1 \mathrm{~mm}$ ). Spatial calibration in Measure option of menu bar or the software, were the step to calibrate images and to ensure the desired unit of measurements, in this case, microns.

In this research, areas of oxidative (slow twitch) and glycolytic (fast twitch) characteristics were important as regions of interest to emphasize by semi-automatic masks of color. This is useful in clinical areas or digital pathology; for example, in the study conducted for Maldjian et al. (2003), by using a software for image analysis of cerebral MRI (magnetic resonance imaging) in which identified regions of interest by masks of color.

Prior to the masks of color was necessary to convert images to grayscale in three channels of primary colors, blue, red and green (RGB channels). The selected channel was the green for its best sharpness to identify the extent of the areas given for morphometric study (Fig. 1). In monochromatic images, scale of color intensities in a pixel recognized by software is from zero (0) to 255 shades of black to white, respectively.

To every image in green channel calibration and automatic color (pseudo color icon in the software) was applied, to evidence oxidative and glycolytic areas in NADH-TR stain (Fig. 2), and low twitch, fast twitch and intermediate twitch fibers in m-ATPase $\mathrm{pH} 4.6$ stain. Each color of masks was specific to each area for morphometric analysis. Areas of intramuscular extracellular connective tissue and white background were excluded for this analysis. Measurements of each area in microns was determined according to the area of color that software assigned in the gray scale intensities.

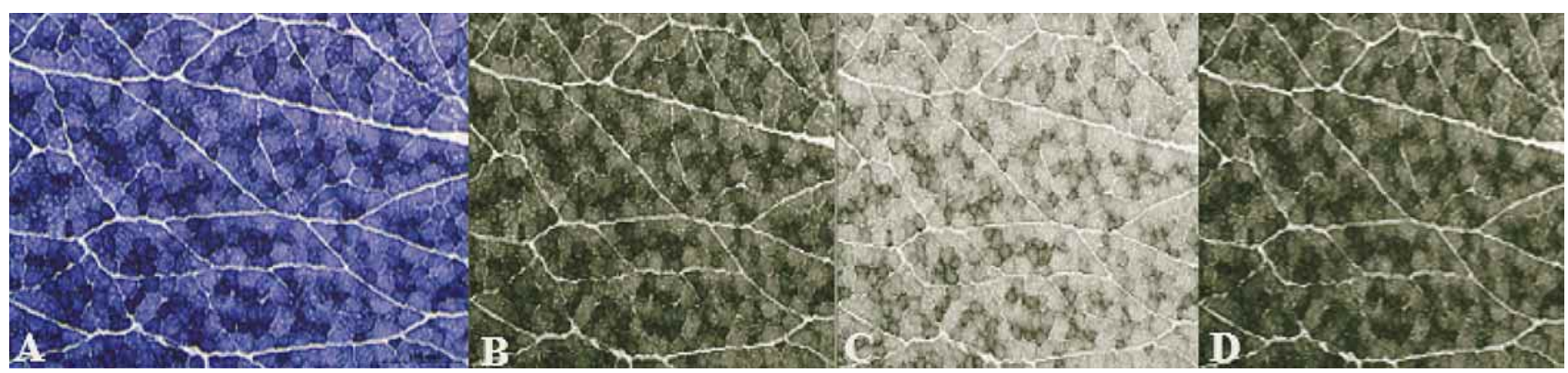

Fig. 1. Extensor carpi radialis longus muscle, control group in NADH-TR staining (A) and grayscale (B, C and D); it is observed different intensity of shades related to the basic colors or channels RGB (red-green- blue). B corresponds to the blue channel, $\mathrm{C}$ the green channel and D, the red one. It is notable in C, histological features of oxidative and glycolytic fibers, similar to original image (A). Magnification 10x. Scale Bar: 100 microns.
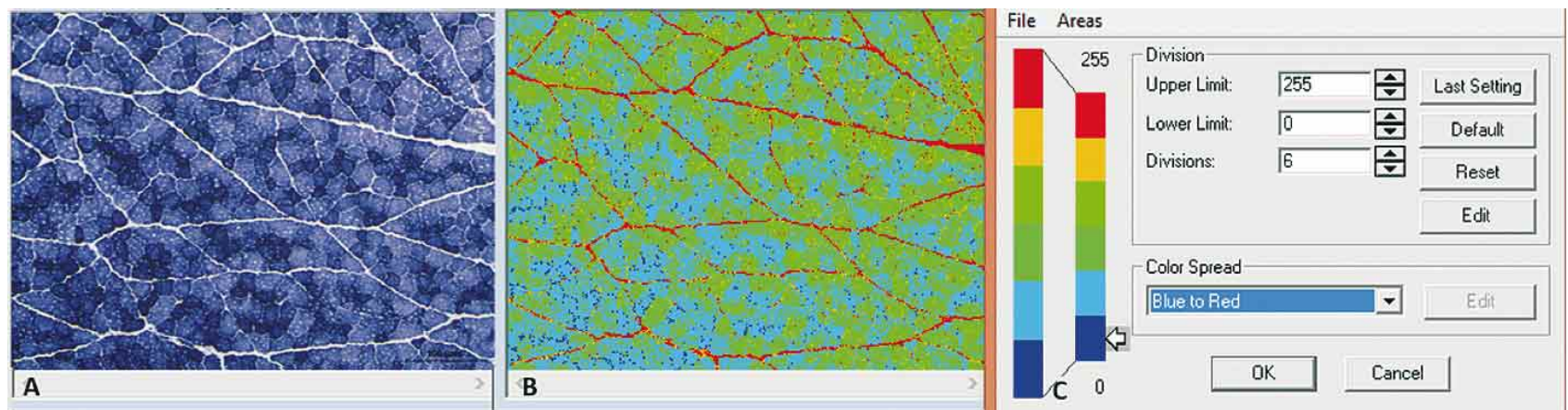

Fig. 2. Image processing. A. Extensor carpi radialis longus muscle, control group in NADH-TR staining. B. Mask of color of regions of interest. Oxidative regions are blue and glycolytic are green. The regions of no interest (extracellular intramuscular matrix) are red. C. The column evidences the gray intensities in which each region is located. The same color does not always indicate the same region of interest or no interest. Magnification 100x. Scale Bar: 100 microns. 
Intraclass Correlation Coefficient. The median of areas were determined to 14 images randomly selected, to apply masks of color. These measurements were acquired among three observers that included two experts in histological characteristics of skeletal muscle tissue and the investigator. Statistical comparison had a concordance coefficient of 0.99 $\mathrm{p}$-value 0.000 . Then, two measurements were carried out to 205 images, randomly selected as well.

Statistical analysis. Kruskal - Wallis test was useful to carry out comparisons of the areas among groups of reperfusion. For comparisons between one and three hours of ischemia during reperfusion, Mann Whitney test was appropiated. Periods that showed significant differences were analyzed by multiple comparisons test, non-parametric Bonferroni post-ANOVA. Non-parametric tests were used due to measurements evidenced high variability among periods of reperfusion, mainly for those that followed three hours of ischemia. SPSS version 22 was the statistical software used for this analysis.

Table I. Control group. Classification of muscle fibers by type of metabolism, NADH-TR, and velocity of contraction, m-ATPase $\mathrm{pH}$ 4.6.

\begin{tabular}{lcc}
\hline & $\begin{array}{c}\text { M. Extens or carpi } \\
\text { radialis longus }\end{array}$ & M. Soleus \\
\cline { 2 - 3 } Oxidative & 59398 & 675890 \\
Glycolytic & $(6.5 \pm 2.8 \%)$ & $(73.2 \pm 1.0 \%)$ \\
& 520662 & 0 \\
Slow twitch & $(59.6 \pm 0.3 \%)$ & --- \\
& 26283 & 114835 \\
Fast twitch & $(6.1 \pm 0.8 \%)$ & $(67.6 \pm 4.6 \%)$ \\
& 229265 & 0 \\
& $(40.0 \pm 0.9 \%)$ & -- \\
\hline
\end{tabular}

Oxidative fibers - Extensor carpi radialis longus - 10X

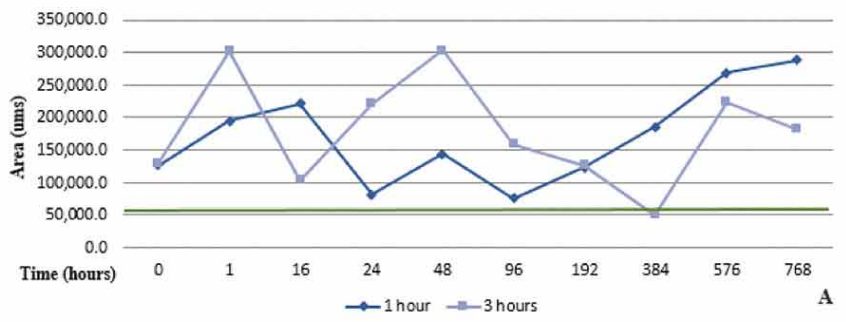

\section{RESULTS}

Control group. Areas of oxidative (slow twitch) and glycolytic (fast twitch) were determined in both histochemical stains, NADH-TR and mATPase. In control group, extensor carpi radialis longus evidenced its normal predominance of glycolytic fast-twitch fibers (Type IIB), while the soleus its oxidative slow-twitch fibers normal pattern (Type I), Table I.

Oxidative fibers and slow twitch fibers. NADH-TR stain in extensor carpi radialis longus, evidenced an increasing in all of the groups of reperfusion in one and three hours of ischemia (Fig. 3). To compare areas among these groups from zero hours to $32 \mathrm{~d}$, the changes were significant for both periods of ischemia, p-value 0.027 for one hour and p-value 0.038 for three hours; mATPase stain in the same muscle, showed lower areas than the control group at one hour of ischemia and reperfusion of zero hours, 16 h, 24 h, two days, four days and eight days (Fig. 4), p-value 0.034 . Figure 5 shows the semiautomatic recognition pattern in this histochemical stain for each type of fiber in this muscle.

The predominance of type of fibers was different in both stains for soleus. Areas observed in NADH-TR decreased in comparison to control (Fig. 3) with important changes in distribution at $16 \mathrm{~h}, 96 \mathrm{~h}$ and $192 \mathrm{~h}, \mathrm{p}$-value 0.026 for one hour and p-value 0.052 for three hours. The comparisons between one and three hours of ischemia and their periods of reperfusion evidenced significant variations in muscle tissues, p-value 0.037. On the other hand, an increasing in mATPase was detected, except in eight days of reperfusion for one hour of ischemia p-value 0.028 , and $16 \mathrm{~d}$ of reperfusion for three hours of ischemia (Fig. 4) p-

Fig. 3. Comparison of the median in the area of oxidative fibers among periods of reperfusion following one hour and three hours of ischemia. The green line indicates control group measures. X-axis: Time; Y: Area. A. The tendency is the increase in the area of oxidative fibers. Note the difference between zero hours and $32 \mathrm{~d}(768 \mathrm{~h})$ of reperfusion, with more variation of values in three hours. B. Fluctuation between areas with a decreased of oxidative fibers in one and three hours of ischemia. Note the difference between periods of reperfusion of one hour (1) and 32 days (768 h). 
Slow twich fibers - Extensor carpi radialis longus - 10x

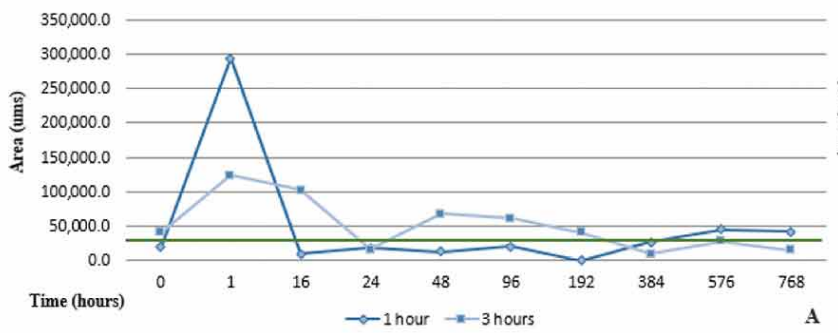

Slow twich fibers. Soleus- $10 x$

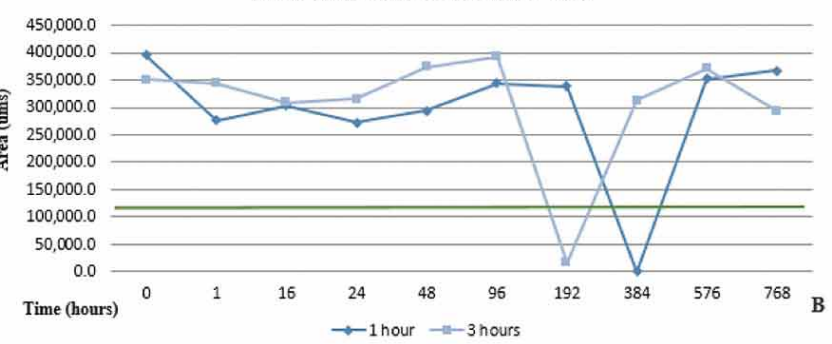

Fig. 4. Comparison of the median in the area of slow twitch fibers among periods of reperfusion following one hour and three hours of ischemia. The green line indicates control group measures. X-axis: Time; Y: Area. A. The tendency is the increase in the area with values near to control in long periods of reperfusion. Areas in one hour and three hours tend to be similar, except for one hour of reperfusion in which values are higher. The differences are among short and long periods of reperfusion. B. Differences were at one-hour ischemia with $8 \mathrm{~d}$ of reperfusion and three hours ischemia and $16 \mathrm{~d}$ of reperfusion; these changes are small among short and long periods.

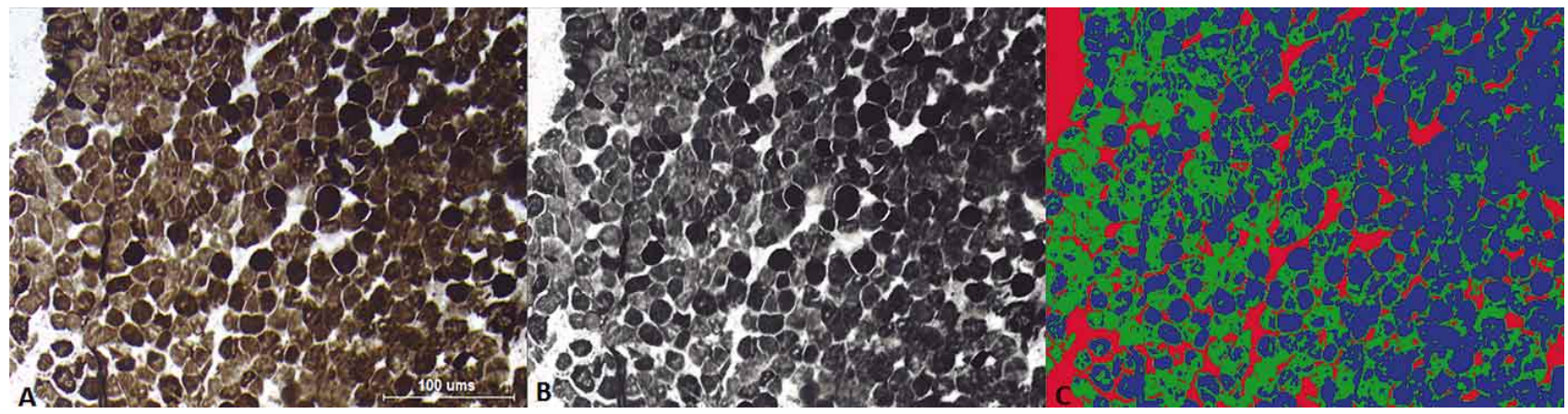

Fig. 5. Extensor carpi radialis longus muscle, ischemia of three hours and reperfusion of one hour in mATPase staining. A. Disorganization pattern evidenced with changes in normal predominance of fiber types. B. Grayscale image in the green channel. C. Mask of color of regions of interest. Slow twitch regions are blue, this region had increased in relation to normal control and fast twitch are green. The regions of no interest are red. Magnification 10x. Scale Bar: 100 microns.

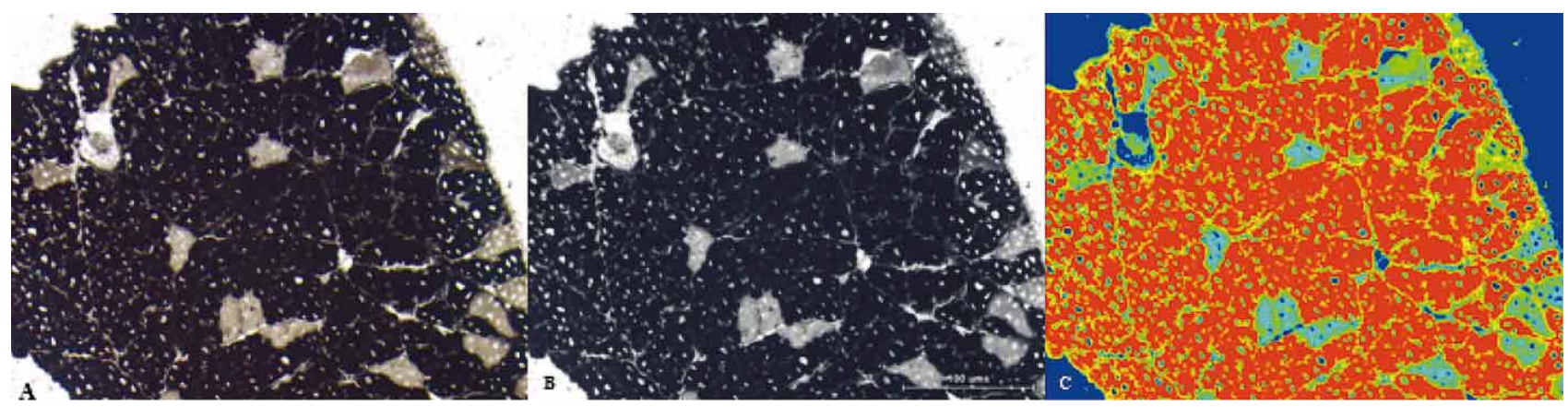

Fig. 6. Soleus muscle, ischemia of one hour-reperfusion of $16 \mathrm{~h}$ in mATPase staining. A. Fast twitch fibers evidenced in the microscopic field. B. Grayscale image in the green channel. C. Mask of color of regions of interest. Slow twitch regions are orange color and fast twitch are light blue. The regions of no interest are dark blue. Magnification 10x. Scale Bar: 100 microns.

value 0.130 , although comparisons of changes among periods of reperfusion were not significant, p value 0.245 . Figure 6 presents the determination of regions of interest in soleus, and Figure 7 the tendency in day 32 of reperfusion.

Glycolytic fibers and fast twitch fibers. The distribution of the areas for extensor carpi radialis longus evidenced a reduction in relation to the control group, this aspect was evidenced for both stains (Figs. 8 and 9). The changes in area of glycolytic fibers among periods of reperfusion were not significant $\mathrm{p}$-value 0.124 for one hour and $\mathrm{p}$-value 0.106 for three hours (Fig. 8). In fast twitch fibers during one hour of ischemia, all areas were lower than the control group, except for zero hour, $16 \mathrm{~h}$ and eight days (Fig. 9), p-value 0.027. In three hours, there were decreasing except at $16 \mathrm{~d}$; variations along reperfusion showed not significant differences, p-value 0.215 . Changes were significant in comparisons between one and three hours of ischemia, p- 


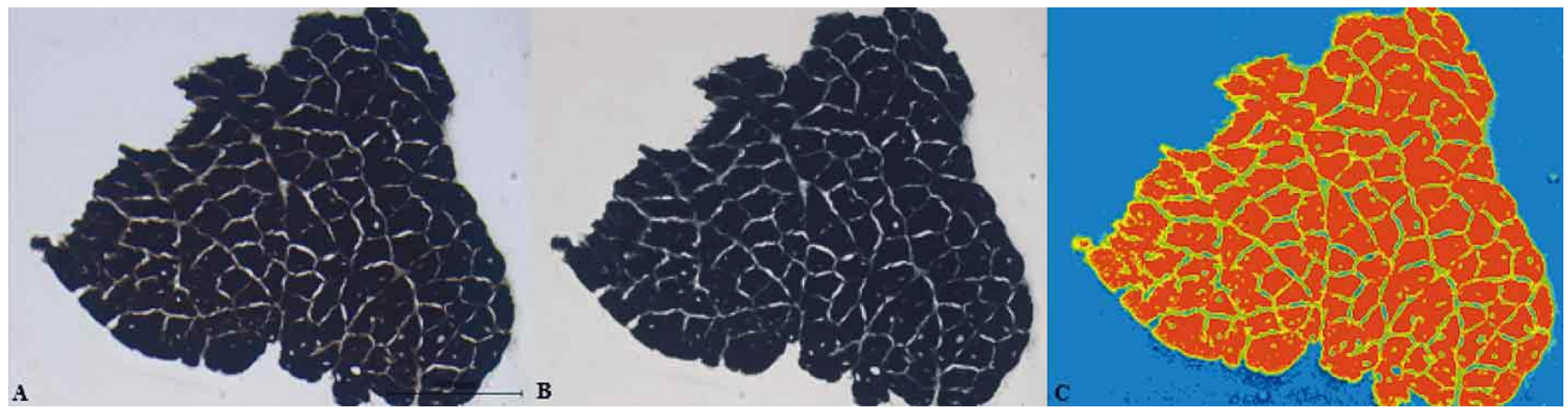

Fig. 7. Soleus muscle, ischemia of three hours-reperfusion of $32 \mathrm{~d}$ in mATPase staining. A. Slow twitch normal tendency evidenced in the microscopic field. B. Grayscale image in the green channel. C. Mask of color of regions of interest. Slow twitch regions are orange color. There is no evidence of intermediate and fast twitch regions. Magnification 10x. Scale Bar: 100 microns.

Glycolytic fibers-Extensor carpi radialis longus-10x

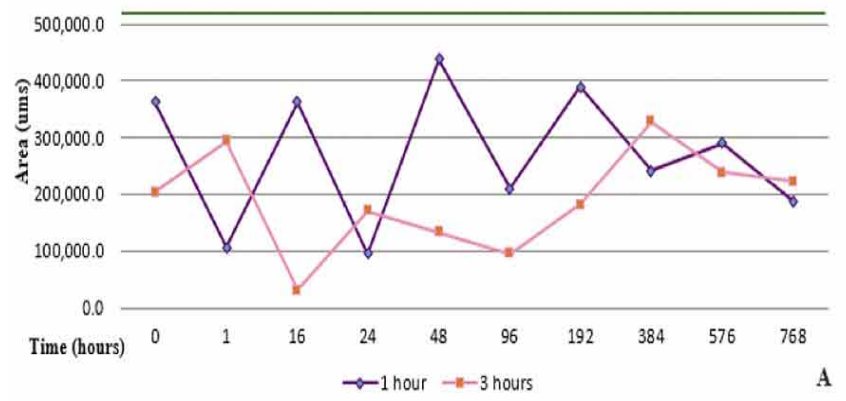

Glycolytic fibers-Soleus-10x

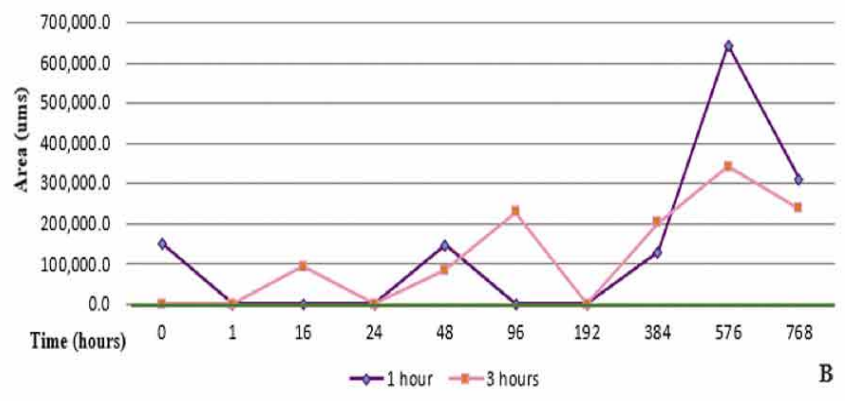

Fig. 8. Comparison of the median in the area of glycolytic fibers among periods of reperfusion following one hour and three hours of ischemia. The green line indicates control group measures. X-axis: Time; Y: Area. A. The distribution of median varies among periods of reperfusion. In ischemia of three hours, there are differences between $16 \mathrm{~h}$ and $16 \mathrm{~d}(384 \mathrm{~h})$ of reperfusion. At $32 \mathrm{~d}(768 \mathrm{~h})$, the area tends to decrease. B. The area increase at the end of the reperfusion. At $32 \mathrm{~d}$, areas are similar in one and three hours of ischemia.

\section{Fast twich fibers-Extensor carpi radialis longus-10x}

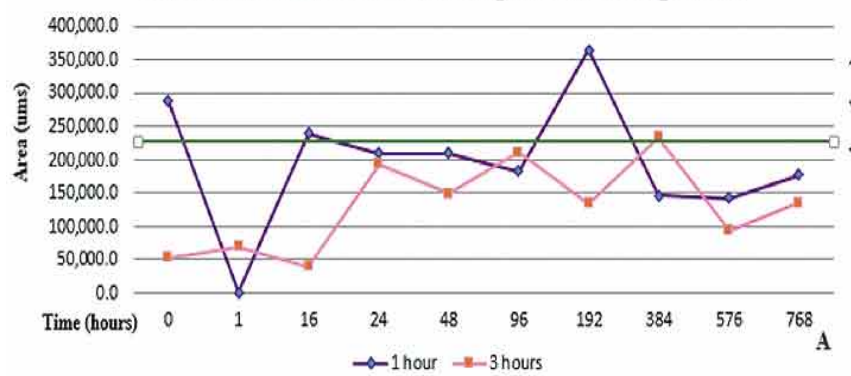

Fast twich fibers-Soleus-10x

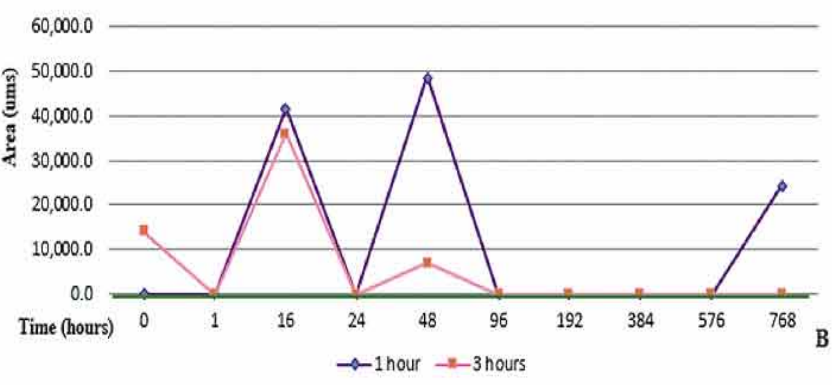

Fig. 9. Comparison of the median in the area of fast twitch fibers among periods of reperfusion following one hour and three hours of ischemia. The green line indicates control group measures. X-axis: Time; Y: Area. A. During reperfusion, differences among one hour and eight days in one-hour ischemia, and $16 \mathrm{~h}$ and $16 \mathrm{~d}$ in three hours of ischemia. B. In both, ischemia of one hour and three hours, just three groups of reperfusion present this kind of fibers, seen in the microscopic field.

value 0.021 , and were noted at one-hour of ischemia and reperfusion of one hour, and three hours of ischemia with reperfusion from $24 \mathrm{~h}$ to $32 \mathrm{~d}$.

The areas for glycolytic fibers of soleus muscle during one hour of ischemia were higher than the normal value at
$16 \mathrm{~h}$, two days and $32 \mathrm{~d}$ (Fig. 8) p-value 0.026. In three hours, there was an increasing in the area at zero $\mathrm{h}, 16 \mathrm{~h}$ and two days p-value 0.050 . Comparing the distribution of fast twitch fibers between one and three hours of ischemia followed by reperfusion, differences were not notable $\mathrm{p}$ value- 0.659 . 


\section{DISCUSSION}

Semiautomatic methods have had positive results with images of ultrasound, tomography and magnetic resonance; in the same way automatic segmentation methods are still being studied (Xian et al., 2015; Mehmood et al., 2013). The applications of semiautomatic segmentation to optical microscopy is one of the fields to research further, in order to recognize elements such as specific types of cells or components of extracellular matrix with more accuracy, as well as color images (Bhattacharyya et al., 2011), for example different histochemistry techniques in histopathology.

In the present study, extensor carpi radialis longus changed the predominance of glycolytic fibers into an oxidative; these areas increased progressively in prolonged periods of reperfusion of $16 \mathrm{~d}(384 \mathrm{~h}), 24 \mathrm{~d}(576 \mathrm{~h})$ and $32 \mathrm{~d}$ (768 h). To glycolytic fibers, the lowest value was in ischemia of three hours and $16 \mathrm{~h}$ of reperfusion, followed by ischemia of one hour and reperfusion of one hour. On day 32 of reperfusion, in one-hour of ischemia the area was $36 \%$ of control, but $43 \%$ in three hours. For fast twitch fibers on the last day of reperfusion, the area was $77 \%$ of normal area in ischemia of one hour, while in three hours of ischemia it was $59 \%$. Chan et al. (2004), compared the response of soleus and vastus that underwent two hours of ischemia, up to six hours of reperfusion. By using immunohistochemical markers for IgM and $\mathrm{C} 3$, found in fast-twitch fibers of vastus a $28 \%$ of injured fibers in comparison to the soleus, which provided $17 \%$ of the affected fibers. During reperfusion injury, type IIB fibers are often affected.

By contrast, in the soleus muscle there was not a drastic change from oxidative to glycolytic during reperfusion. This predominantly oxidative muscle endured better reperfusion injury. The area of oxidative fiber observed in NADH-TR staining, was generally lower than that measured in control. The smaller area of zero microns was in one hour of ischemia with reperfusion of two days, and three hours of ischemia with reperfusion of four days and $32 \mathrm{~d}$. In one hour of ischemia and reperfusion of $32 \mathrm{~d}$, the area was only $21 \%$ of the established as normal. For glycolytic fibers, medians were higher than the control. The largest proportion was in ischemia of one hour and reperfusion of $24 \mathrm{~d}$. At $32 \mathrm{~d}$, the values remained higher than the control and could be considered as evidence of the ability of adaptation of muscle to ischemia and reperfusion injury. By mATPase $\mathrm{pH} 4.6$ staining for soleus, the presence of fast twitch fibers was evident from zero hours of reperfusion and for the rest of periods, for both, one hour and three hours of ischemia; this type of fibers decreased progressively and the muscle recovered its predominance of slow twitch fibers on day 32 of reperfusion (Fig. 7).

Previous studies conducted by Woitaske \& McCarter and Walters et al., exposed that fiber type, influences the adaptive changes of the predominantly glycolytic muscles by day 14 of reperfusion. In this research, injuries were reversible for extensor carpi radialis longus and soleus, which evidenced partial restoration at $32 \mathrm{~d}$.

The percentage of areas of the fiber types in mATPase pH 4.6 stain obtained in this study, were similar to that described by other authors (Table II). Although measurement techniques were different, values approached each other. Soukup et al., carried out cell counting by using stereology; Pullen (1977) performed direct counting of each type of fiber per microscopic field. In this research, image analysis was performed by measurements of areas based on regions of interest; this is as a practical and convenient method to recognize the specific zone to be evaluated. The main advantage of this technique is the minor manipulation of images during image processing.

Comparisons of measurements by using correlation coefficient among data published by other authors and data of the present research, evidence for extensor carpi radialis longus a good correlation of 0.76 , and a strong correlation of 0.98 for soleus. This confirms the accuracy of the method to detect alterations in tissues respect to a normal pattern. Histochemical stains and semiautomatic measurements are low cost techniques to be applied in a formal way in clinical practice, for example, in the pathological analysis of diseases. According to Chieco et al. (2013), a complete automatic analysis in tissues could represent bias, as many software do not recognize differences among cells and structures in the same microscopic field. That is why the expertise of histologist, pathologist or investigator, is another element of the analysis, thus the recognition patterns happen to be semi-automatic.

Table II. Comparison of normal distribution of fiber types by staining mATPase in muscles of Wistar rat.

\begin{tabular}{llllccc}
\hline Reference / Type of fiber & \multicolumn{2}{c}{ M. Extensor carpi radialis longus (\%) } & \multicolumn{3}{c}{ M. Soleus (\%) } \\
\cline { 2 - 7 } & Type I & Type IIA & Type IIB & Type I & Type IIA & Type IIB \\
\hline Soukup et al. (1979) & 4.5 & 27.8 & 35.1 & 76 & 25.6 & 0 \\
Pullen et al. (1977) & 9.6 & 51.2 & 39.3 & 84 & 23.7 & 0 \\
Soukup et al. (2002) & 5.5 & 18.8 & 75.5 & 96.1 & $*$ & 3.9 \\
Actual rese arch & 6.1 & 53.7 & 40.0 & 67.6 & 32.3 & 0 \\
\hline
\end{tabular}




\section{CONCLUSIONS}

In both muscles, changes in the distribution of normal areas were present even at 32 days (768 h) of reperfusion. Periods of 16 h, 24 h, two days, four days and eight days were identifying as relevant in one and three hours of ischemia. The extensor carpi radialis longus tolerated three hours of ischemia with mild changes in its histological constitution; however, distribution of fibers evidenced variations in early hours of reperfusion. On the other hand, soleus tolerated reperfusion and presented gradual recovering. Although skeletal striated muscle tissue has a strong ability to adapt to injury, in some cases, this tissue does not recover its complete morphological and functional characteristics.
Image analysis techniques as semiautomatic measurements of regions of interest have applications to clinical situations that can be used and associated with knowledge of experts (radiologist, investigators or pathologist), to carry out a more precise criteria of observation and clinical analysis of abnormal characteristics of structures, organs and tissues. These are aspects in which image analysis techniques must evolve.

ACKNOWLEDGEMENTS. Author wants to acknowledge to Teblami (soft and mineralized tissues) Investigation Group/Universidad del Valle, Nhora Holguín, Martha Ceballos and Prof. Liliana Flórez MSc. for their valuable contributions to this work.

ROSERO-SALAZAR, D. H. Análisis de imágenes de fibras musculares oxidativas y glucolíticas durante la reperfusión mediante segmentación basada en regiones. Int. J. Morphol., 34(1):127-135, 2016.

RESUMEN: Diferentes situaciones causan isquemia y reperfusión afectando los tejidos que se encuentran por debajo del nivel de lesión. En esta investigación, se determinaron las características anormales en la distribución de los tipos de fibras musculares en los músculos sóleo y extensor radial longo del carpo, durante períodos cortos de isquemia y de reperfusión tanto cortos como prolongados. Las fibras se clasificaron mediante las técnicas de tinción de histoquímica enzimática NADH-TR y miosina-ATPasa. Las mediciones de las áreas realizadas mediante procesamiento de imágenes y el uso de la segmentación basada en regiones, evidenciaron cambios significativos en la distribución durante la reperfusión seguida a una y tres horas de isquemia, así como en las comparaciones para todos los períodos de reperfusión. Este estudio es un aporte en la evidencia sobre el uso de procedimientos prácticos de análisis de imágenes en el diagnóstico de alteraciones tisulares.

PALABRAS CLAVE: Histoquímica; Isquemia; Reperfusión; Análisis de imágenes; Segmentación.

\section{REFERENCES}

Bhattacharyya, S.; Maulik, U. \& Dutta, P. Multilevel image segmentation with adaptive image context based thresholding. J. Appl. Soft Comput., 11(1):946-62, 2011.

Carmo-Araújo, E.; Dal-Pai-Silva, M.; Dal-Pai, V.; Cecchini, R. \& Anjos Ferreira, A. L. Ischaemia and reperfusion effects on skeletal muscle tissue: morphological and histochemical studies. Int. J. Exp. Pathol., 88(3):147-54, 2007.

Chan, R. K.; Austen, W. G. Jr.; Ibrahim, S.; Ding, G. Y.; Verna, N.; Hechtman, H. B. \& Moore, F. D. Jr. Reperfusion injury to skeletal muscle affects primarily type II muscle fibers. J. Surg. Res., 122(1):54-60, 2004.

Chieco, P.; Jonker, A.; De Boer, B. A.; Ruijter, J. M. \& Van Noorden, C. J. Image cytometry: protocols for 2D and 3D quantification in microscopic images. Prog. Histochem. Cytochem., 47(4):211-333, 2013.
Corona, B. T. \& Rathbone, C. R. Accelerated functional recovery after skeletal muscle ischemia-reperfusion injury using freshly isolated bone marrow cells. J. Surg. Res., 188(1):100-9, 2014.

de Freitas, C. E. A.; Zambelli Freitas, S. B.; da Silva Lopes, F.; Pai-Silva, M. D. \& da Cruz Piçarro, I. Skeletal muscles with antagonistic muscular actions: Morphological, contractile and metabolic characteristics. Int. J. Morphol., 27(4):1173-8, 2009.

Gueguen, N.; Lefaucheur, L.; Fillaut, M. \& Herpin, P. Muscle fiber contractile type influences the regulation of mitochondrial function. Mol. Cell. Biochem., 276(1-2):15-20, 2005.

Hebling, A.; Scabora, J. E., \& Marretto Esquisatto, M. A. M. Muscle fibre types and connective tissue morphometry in frontal muscle of Norfolk rabbits (Oryctolagus cuniculus). Int. J. Morphol., 27(1):187-91, 2009. 
Hebling, A.; Esquisatto, M. A. M. \& Lima Castro, H. A. Histochemical study of enzyme in frontal muscle of Norfolk rabbit (Oryctolagus cuniculus). Int. J. Morphol., 24(2):1514, 2006.

Hocker, A. D.; Boileau, R. M.; Lantz, B. A.; Jewett, B. A.; Gilbert, J. S. \& Dreyer, H. C. endoplasmic reticulum stress activation during total knee arthroplasty. Physiol. Rep., 1(3):e00052, 2013.

Kalmar, B.; Blanco, G. \& Greensmith, L. Determination of Muscle Fiber Type in Rodents. Curr. Protoc. Mouse Biol., 2(3):231-43, 2012.

Latorre, R.; Gil, F.;Vázquez, J. M.; Moreno, F.; Mascarello, F. \& Ramirez, G. Skeletal muscle fibre types in the dog. J. Anat., 182(Pt. 3):329-37, 1993.

Lexell, J.; Jarvis, J. C.; Currie, J.; Downham, D. Y. \& Salmons, $\mathrm{S}$. Fibre type composition of rabbit tibialis anterior and extensor digitorum longus muscles. J. Anat., 185(Pt. 1):95-101, 1994.

Maldjian, J. A.; Laurienti, P. J.; Kraft, R. A. \& Burdette, J. H. An automated method for neuroanatomic and cytoarchitectonic atlas-based interrogation of fMRI data sets. Neuroimage, 19(3):1233-9, 2003.

Mehmood, I.; Ejaz, N.; Sajjad, M. \& Baik, S. W. Prioritization of brain MRI volumes using medical image perception model and tumor region segmentation. Comput. Biol. Med., 43(10):1471-83, 2013.

Pullen, A. H. The distribution and relative sized of fibre types in the extensor digitorum longus and soleus muscles of the adult rat. J. Anat., 123(Pt. 2):467-86, 1977.

Purslow, P. P. Muscle fascia and force transmission. J. Bodyw. Mov. Ther., 14(4):411-7, 2010.

Scott, W.; Stevens, J. \& Binder-Macleod, S. A. Human skeletal muscle fiber type classifications. Phys. Ther., 81(11):18106, 2001.

Silveira, L. R.; Pereira-Da-Silva, L.; Juel, C. \& Hellsten, Y. Formation of hydrogen peroxide and nitric oxide in rat skeletal muscle cells during contractions. Free Radic. Biol. Med., 35(5):455-64, 2003.

Soukup, T.; Zacharová, G. \& Smerdu, V. Fibre type composition of soleus and extensor digitorum longus muscles in normal female inbred Lewis rats. Acta Histochem., 104(4):399-405, 2002.

Vivo, J.; Morales, J. L.; Diz, A.; Miró, F.; Galisteo, A. M. \& Agüera, E. Estudio morfológico y morfométrico del músculo oblicuo dorsal del perro. Arch. Med. Vet., 37(1):49-54, 2004.
Walters, T. J.; Kragh, J. F. \& Baer, D. G. Influence of fiber-type composition on recovery from tourniquet-induced skeletal muscle ischemia-reperfusion injury. Appl. Physiol. Nutr. Metab., 33(2):272-81, 2008.

Woitaske, M. D. \& McCarter, R. J. Effects of fiber type on ischemiareperfusion injury in mouse skeletal muscle. Plast. Reconstr. Surg., 102(6):2052-63, 1998.

Xian, M.; Zhang, Y. \& Cheng, H. D. Fully automatic segmentation of breast ultrasound images based on breast characteristics in space and frequency domains. J. Pattern Recognit., 48(2):48597, 2015.

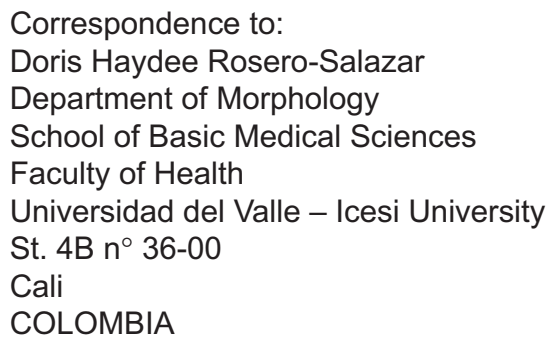

Email: dorhaross@gmail.com

Received: 30-06-2015

Accepted: 19-10-2015 